\begin{tabular}{|c|c|}
\hline Title & On the Electronic Structure Origin of Mechanochemically Induced Selectivity in A cid-Catalyzed Chitin Hydrolysis \\
\hline Author(s) & De Chavez, Danjo; Kobay ashi, Hirokazu; Fukuoka, A tsushi; Hasegawa, Jun-ya \\
\hline Citation & $\begin{array}{l}\text { The Journal of Physical Chemistry A, 125(1), 187-197 } \\
\text { https://doi.org/10.1021/acs.jpca.0c09030 }\end{array}$ \\
\hline Issue Date & 2021-01-14 \\
\hline Doc URL & http://hdl.handle.net/2115/83829 \\
\hline Rights & $\begin{array}{l}\text { This document is the A ccepted Manuscript version of a Published Work that appeared in final form in [The Journal of } \\
\text { Phy sical Chemistry A ], copyright @ } \mathrm{A} \text { merican Chemical Society after peer review and technical editing by the } \\
\text { publisher. To access the final edited and published work see http://pubs.acs.org/articlesonrequest/A OR- } \\
\text { K284U GHTFY DHZCT GDF7M }\end{array}$ \\
\hline Type & article (author version) \\
\hline Additional Information & There are other files related to this item in HUSCAP. Check the above URL. \\
\hline File Information & MS_Nonhighlighted_20201211.pdf \\
\hline
\end{tabular}

Instructions for use 


\title{
On the Electronic Structure Origin of
}

\section{Mechanochemically Induced Selectivity in Acid Catalyzed Chitin Hydrolysis}

\author{
Danjo De Chavez, ${ }^{\dagger}$ Hirokazu Kobayashi, ${ }^{\ddagger}$ Atsushi Fukuoka, ${ }^{\ddagger}$ and Jun-ya \\ Hasegawa*, \\ †Graduate School of Chemical Sciences and Engineering, Hokkaido University, N13W8, \\ Kita-Ku, Sapporo 060-0810, Japan \\ †Institute for Catalysis, Hokkaido University, N21W10, Kita-Ku, Sapporo 001-0021, Japan \\ E-mail: hasegawa@cat.hokudai.ac.jp
}




\begin{abstract}
Recently, mechanical ball milling was applied to chitin depolymerization. The mechanical activation afforded higher selectivity toward glycosidic bond cleavage over amide bond breakage. Hence, the bioactive $N$-acetylglucosamine (GlcNAc) monomer was preferentially produced over glucosamine. In this regard, the force-dependent mechanochemical activation-deactivation process in the relaxed and pulled GlcNAc dimer undergoing deacetylation and depolymerization reactions was studied. For the relaxed case, the activation energies of the rate-determining steps (RDS) proved that the two reactions could occur simultaneously. Mechanical forces associated with ballmilling was approximated with linear pulling and was introduced explicitly in the RDS of both reactions through force modified potential energy surfaces (FMPES) formalism. In general, as the applied pulling force increases, the activation energy of the RDS of deacetylation shows no meaningful change, while that of depolymerization decreases. This result is consistent with the selectivity exhibited in the experiment. Energy and structural analyses for the depolymerization showed that the activation can be attributed to a significant change in the glycosidic dihedral at the reactant state. A lone pair of the neighboring pyranose ring $\mathrm{O}$ adopts a syn-periplanar conformation relative to the glycosidic bond. This promotes electron donation to the $\sigma^{*}$-orbital of the glycosidic bond, leading to activation. Consequently, the Brønsted-Lowry basicity of the glycosidic oxygen also increases, which can facilitate acid catalysis.
\end{abstract}

\title{
Introduction
}

Chitin, one of the abundant naturally available carbohydrates, is a polymer of $N$-acetylglucos -amine linked by $\beta$-glycosidic linkages. The said biopolymer is conventionally derived from the base digestion of the exoskeleton of arthropods, such as crabs and shrimps, ${ }^{1,2}$ followed by deproteination using alkaline proteases and proteolytic bacteria. ${ }^{3-5}$

The chitin monomer, $\mathrm{N}$-acetylglucosamine, is typically obtained via acid hydrolysis of the polymer. Unfortunately, the acid-catalyzed hydrolysis of chitin produces two major products: 
$N$-acetylglucosamine and glucosamine. ${ }^{6}$ Glucosamine results from $N$-deacetylation during the depolymerization.

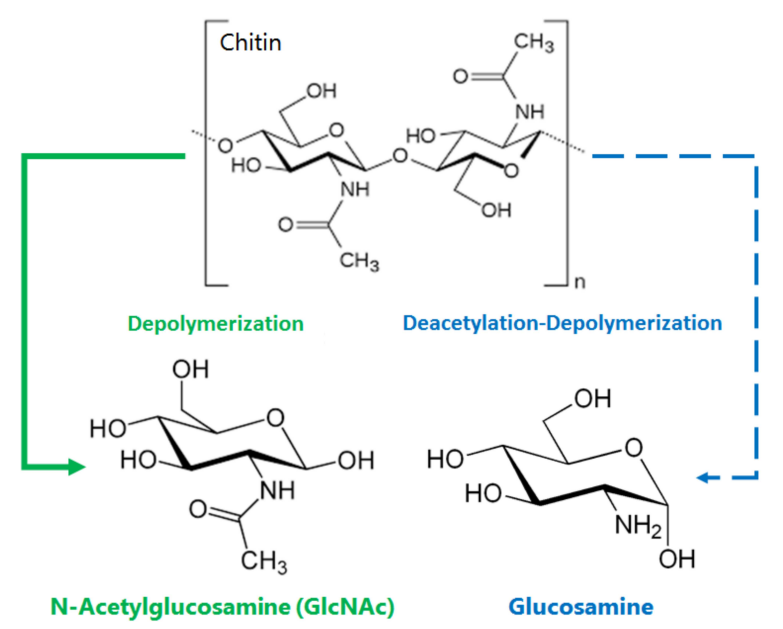

Figure 1: Possible chitin hydrolysis products. Glucosamine is derived from the hydrolysis of the acetyl group. $N$-acetylglucosamine is obtained by the hydrolysis of the glycosidic bond.

The structures of chitin, $N$-acetylglucosamine and glucosamine are shown in Figure 1. Of the two possible products, $\mathrm{N}$-acetylglucosamine is of higher value in comparison to glucosamine. The value of $N$-acetylglucosamine lies to its diverse utility. It has been used as a starting material in the synthesis of N-containing compounds. ${ }^{7}$ Furthermore, $\mathrm{N}$ acetylglucosamine is a bioactive molecule that plays a role in cell signaling. ${ }^{8}$ The pharmacological activities of this molecule have also been proven, including inflammation inhibition activity and a potential anti-cancer activity. ${ }^{9,10}$ Hence, selective depolymerization is desired for industrial purposes. In conventional processes, a high concentration of acid catalyst is employed to achieve a significant selectivity toward the desired $N$-acetylglucosamine product which produces high acid wastes.

Recently, ball milling was employed to acid-impregnated chitin to increase the selectivity toward depolymerization. ${ }^{11}$ This process afforded the desired selectivity with significantly low acid catalyst requirement. A theoretical study on mechanochemical activation of lignin was investigated by East and colleagues. ${ }^{12}$ Ball milling is a preparative grinding technique which falls under the mechanochemical paradigm. Mechanochemistry can be defined as the 
interaction of external mechanical forces with a chemical system. An in-depth review on the theoretical aspects of mechanochemistry was written by Marx and Ribas-Arino, ${ }^{13}$ and more recently, by Stauch and Dreuw. ${ }^{14}$ In comparison to the different modes of activation, such as the thermochemical activation and photochemical activation, mechanochemistry activates chemical reactions using a force vector. A typical example is single-molecule forcemediated activation in atomic force microscopy (AFM) experiments. ${ }^{14-16}$ As this technique allows a specific part of the atom to be pulled in a specific direction, the translation in the computational approach is direct and intuitive. In comparison, techniques such as ball milling, apply forces in a random fashion. Friction, compression, and shearing are also associated with ball milling. In addition to these, the impact of the ball is expected to increase temperature for minute instance. Therefore, an exact quantum chemical simulation has not been conducted.

Several fundamental methods have been developed to calculate the potential energy surface under an external force, such as COGEF (constrained geometries simulate external force), ${ }^{17}$ FMPES (force-modified potential energy surface), ${ }^{18,19}$ EFEI (external force is explicitly included), ${ }^{20}$ and EGO (enforced geometry optimization). ${ }^{21}$ However, the complexity of simulating ball milling experiments hindered mechanistic calculations for related phenomena. A fundamental chemical understanding of this phenomenon is necessary to guide the further development of related processes. In this regard, the effect of ball milling on chitin hydrolysis reactions has been studied via theoretical simulations. This work is focused on the electronic structure understanding of the mechanochemical causality of selectivity in acid-catalyzed chitin hydrolysis under an external force. Furthermore, this work offers a simplified approach to treating forces for ball milling simulations. 


\section{Methods}

Density functional theory (DFT) calculations were conducted to investigate the depolymerization selectivity afforded by ball milling in competing chitin hydrolysis reactions. B3LYP was selected as the exchange-correlation functional. ${ }^{22}$ All calculations were carried using 6-311G(d,p) basis sets. ${ }^{23}$ The chitin dimer was modeled using a $N$-acetylglucosamine (GlcNAc) dimer. The solvation model used in this study is a mix of implicit and explicit water environments. This mixed model was successfully applied to calculate the potential energy surface of the acid-catalyzed hydrolysis of cellobiose. ${ }^{24}$ A total of 15 water molecules was introduced around the GlcNAc dimer. In addition, a polarizable continuum model (PCM $)^{25-27}$ with the dielectric constant of water (78.3553) was used. To simulate the acidic environment in the experiments, a singly protonated system $(+1)$ was adopted.

The depolymerization and deacetylation reaction mechanisms were examined step-wise. Potential energy profiles were calculated with the computational setting described above. Transition states were verified using frequency and intrinsic reaction coordinate calculations. ${ }^{28}$ Natural population analysis was utilized for partial charge determinations. Donoracceptor orbital interactions were analyzed using second-order perturbation theory analysis of the Fock matrix in the natural bonding orbital (NBO) basis. ${ }^{29-34}$

The mechanical forces applied by ball milling were simulated using explicit addition of pulling forces to selected atoms in the dimer through the force modified potential energy surface (FMPES) formalism. ${ }^{18}$ The multi-directional forces associated with the impact of the ball were simplified to linear pulling. That is, a single effective resultant vector can be derived from the variety of mechanical forces experienced by molecules due to ball milling. The forces given by the ball with respect to the molecular plane can be considered as coplanar or non-coplanar. The non-coplanar forces can be attributed to mainly break and reform interchain hydrogen bonding which can result to amorphization in experiments. ${ }^{35}$ Coplanar forces would exist as a set of radially outward vector from point of impact as shown in Figure S1. Since these vectors are additive, there exist a resultant linear vector passing through the 


\section{Pulling Schematic}

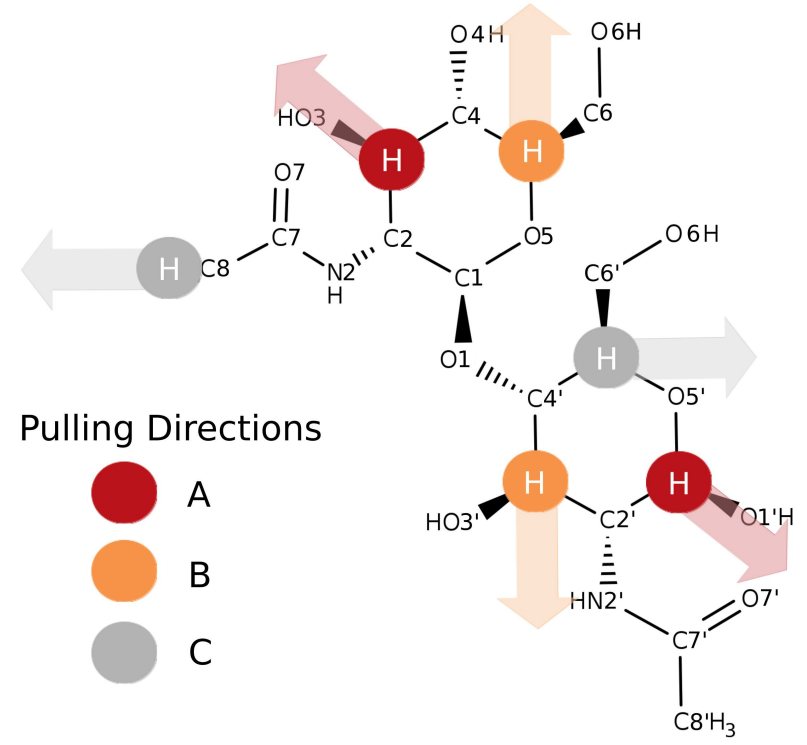

Figure 2: Three sets (A,B, and C) of the pulling directions (arrows) and pulling points (circles) in the model structure

molecular model. Hence, linear pulling can approximate ball milling. In this study, three pulling coordinates were sampled in the rate-determining steps (RDS) of the competing hydrolysis reactions. The pulling scheme is illustrated in Figure 2. Pulling direction A is along the glycosidic linkage of the polymer chain, Pulling Direction B samples with a direction force vector which is non-parallel to both hydrolyzable groups, while Pulling Direction $\mathrm{C}$ includes the hydrolyzable acetyl group, which captures the effect of other pulling coordinates concerning the elongation of $\mathrm{C}-\mathrm{N}$ bond in the RDS of the deacetylation reaction. In all cases, hydrogen atoms were pulled because of the stability of $\mathrm{C}-\mathrm{H}$ bond against pulling. It was observed that in the limits of the sampled force magnitude and directions, the pulled hydrogen atoms remained intact and were not broken in a purely mechanical manner; hence, the application of forces in $\mathrm{C}-\mathrm{H}$ bonds to study depolymerization and deacetylation is a justifiable approach.

To introduce the pulling forces during geometry optimizations, the Gaussian $09^{36}$ optimization cycle was modified. This in-house version executes the algorithm given in Figure 3 using the External function of G09. Process A is the parent G09 process and determines the 


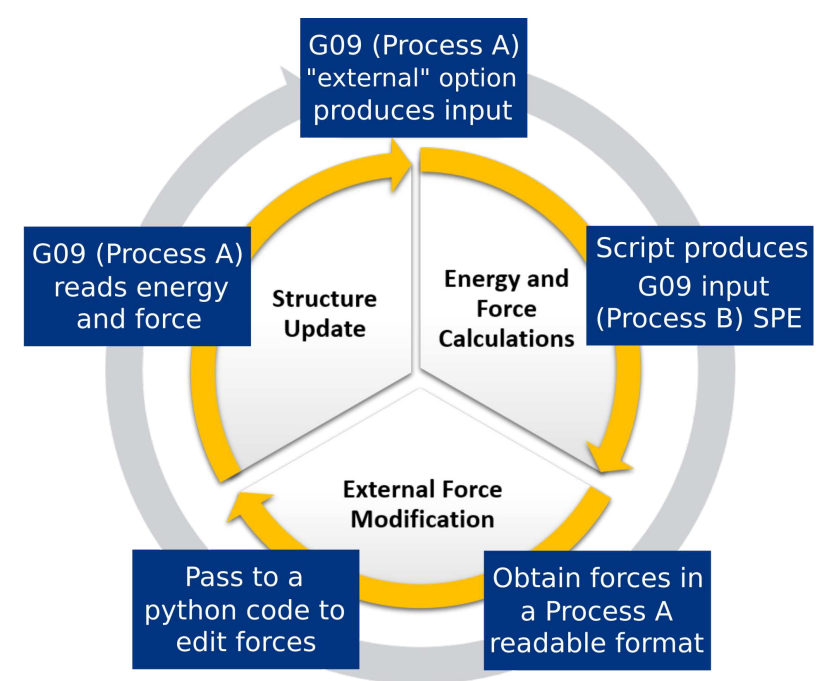

Figure 3: Force modification algorithm. SPE means single point energy calculation

progression of the geometry optimizations. Process B is the daughter G09 process and serves as an external quantum chemical calculator for atomic forces and energies. Before process B passes atomic coordinates, energy, and forces to process A, an external force is added to the force of the pulling points by a python code. The addition of an external force vector to the calculated nuclear atomic force components effectively includes the effect of pulling along the selected directions. A more detailed description of the FMPES implementation is included in SI.

\section{Results \& Discussion}

There are two possible hydrolysis reactions of chitin, both of which can be catalyzed by an acid: (1) deacetylation and (2) depolymerization. The mechanisms of these two reactions are illustrated in Figure 4.

Deacetylation starts with the protonation of the carbonyl oxygen, which increases the positive charge of the carbonyl carbon (A_Int1). This is followed by a nucleophilic attack of water (A_Int2). It was found that the incoming water molecule simultaneously loses a proton, which was also determined by Zahn and co-workers in another theoretical study. ${ }^{37,38}$ 


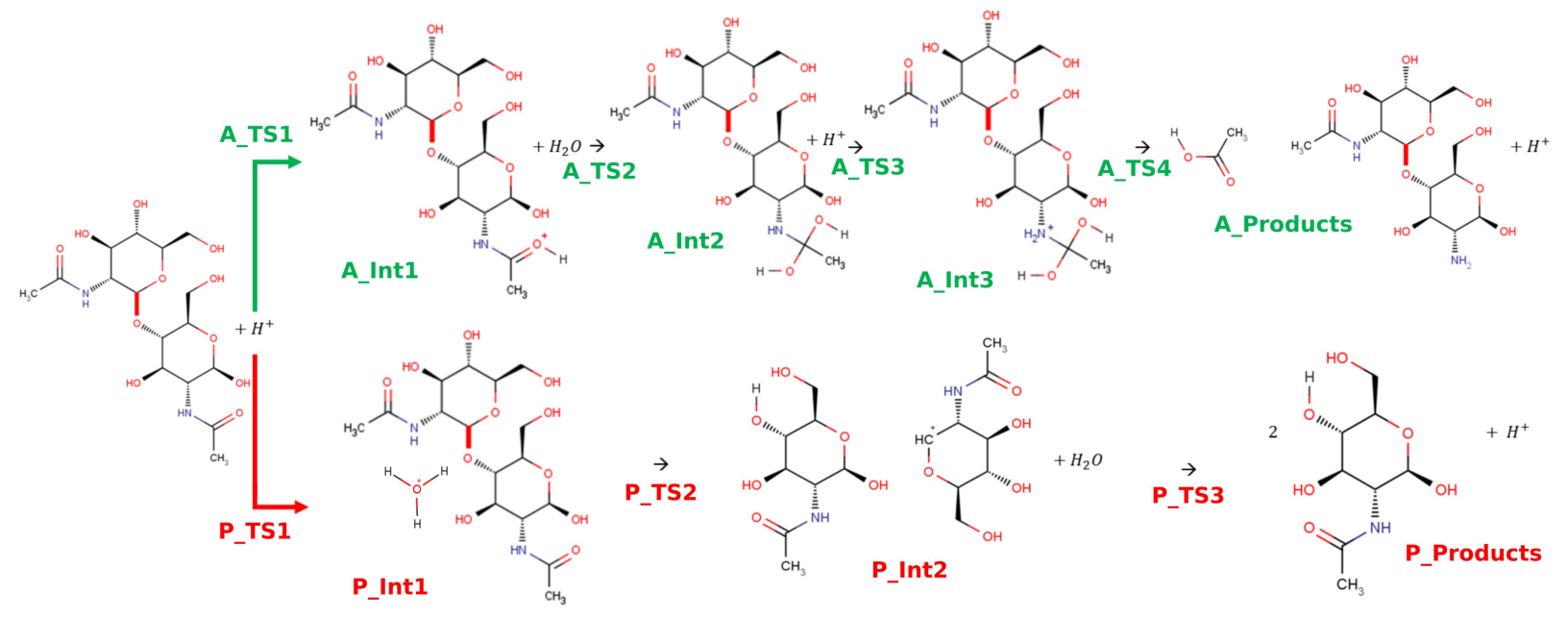

Figure 4: Acid-catalyzed deacetylation (green) and depolymerization (red) reaction mechanisms. (A - deacetylation, P - depolymerization, Int - intermediate and TS - transition state)
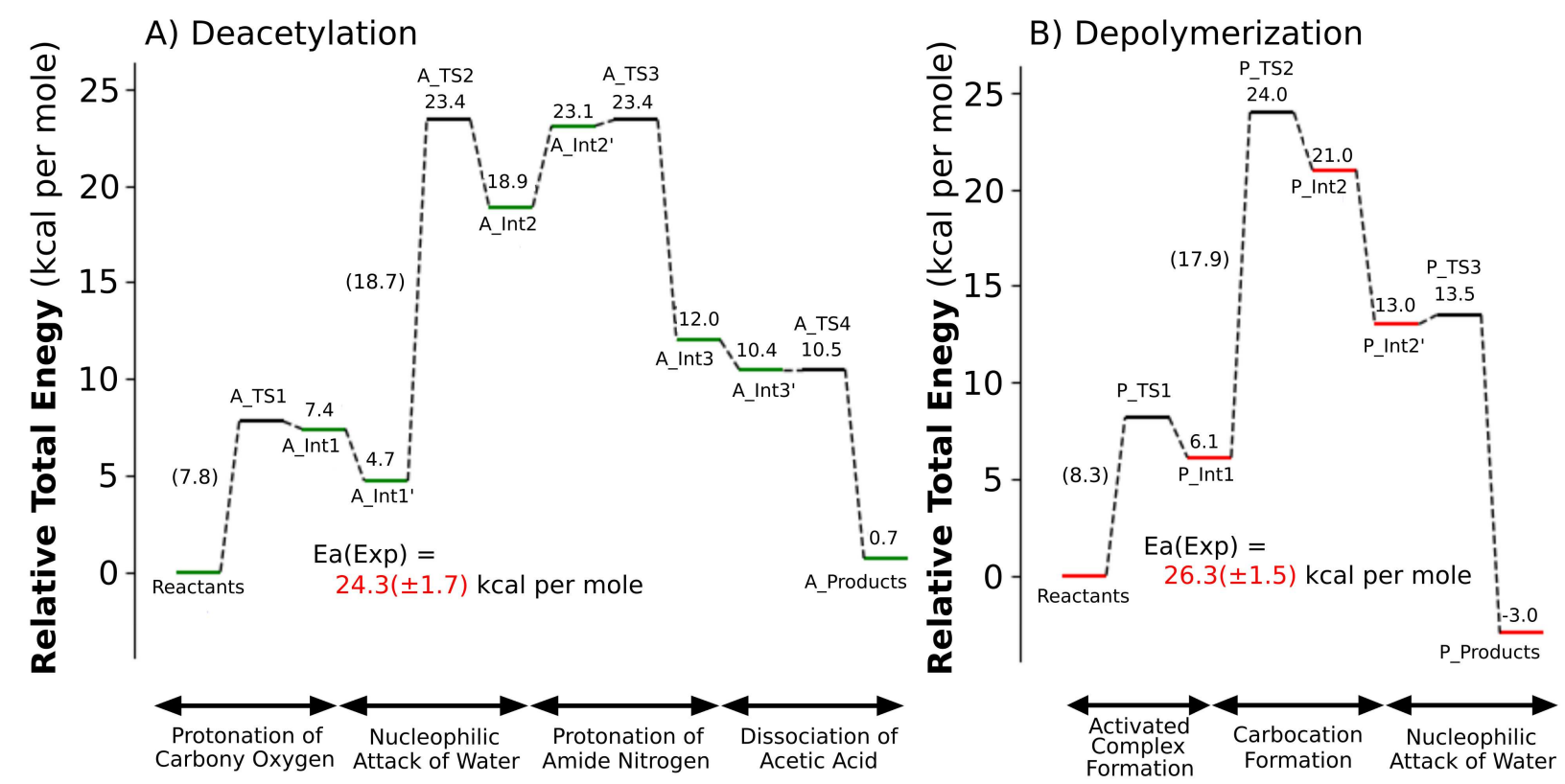

Figure 5: Reaction mechanism energy diagrams of (A) deacetylation and (B) depolymerization. Activation energies are shown in parentheses. 
Proton transfer to the amide nitrogen follows (A_Int3), after which acetic acid is dissociated (A_Products).

Depolymerization is led by the hydrolysis of the glycosidic bond. This is initiated by the protonation of the glycosidic oxygen, which simultaneously breaks the glycosidic bond producing a carbocation (P_Int2). ${ }^{39}$ To complete the reaction, a solvent water molecule attacks the carbocation to form two GlcNAc products (P_Products).

The energy diagrams for the deacetylation and depolymerization are shown in Figure 5. The RDSs were determined as the elementary reaction step with the highest energy requirement. For the deacetylation reaction, the nucleophilic attack of water was determined to be the RDS with an activation energy $\left(E_{a}\right)$ of $18.7 \mathrm{kcal} \cdot \mathrm{mol}^{-1}$. Conversely, the formation of the carbocation was considered as the RDS of the depolymerization reaction with an $E_{a}$ of $17.9 \mathrm{kcal} \cdot \mathrm{mol}^{-1}$. In the experiments, these steps were also determined as the RDSs of the respective reactions. The calculated values are comparable to the experimental activation energies which were $24.3( \pm 1.7) \mathrm{kcal} \cdot \mathrm{mol}^{-1}$, and $26.3( \pm 1.5) \mathrm{kcal} \cdot \mathrm{mol}^{-1}$ respectively. ${ }^{6}$ The relative energies of A_TS2 $\left(23.4 \mathrm{kcal} \cdot \mathrm{mol}^{-1}\right)$ and P_TS2 $\left(24.0 \mathrm{kcal} \cdot \mathrm{mol}^{-1}\right)$ could be regarded as apparent activation energies. These values are close to the experimental ones. As revealed by the calculated $E_{a} s$ of the corresponding RDSs, the two reactions could occur simultaneously due to the similar barrier heights. The results also correspond to the experimental results, indicating the loss of the high value product which is $N$-acetylglucosamine. The optimized structures for the RDS of the depolymerization and deacetylation are included in SI. 


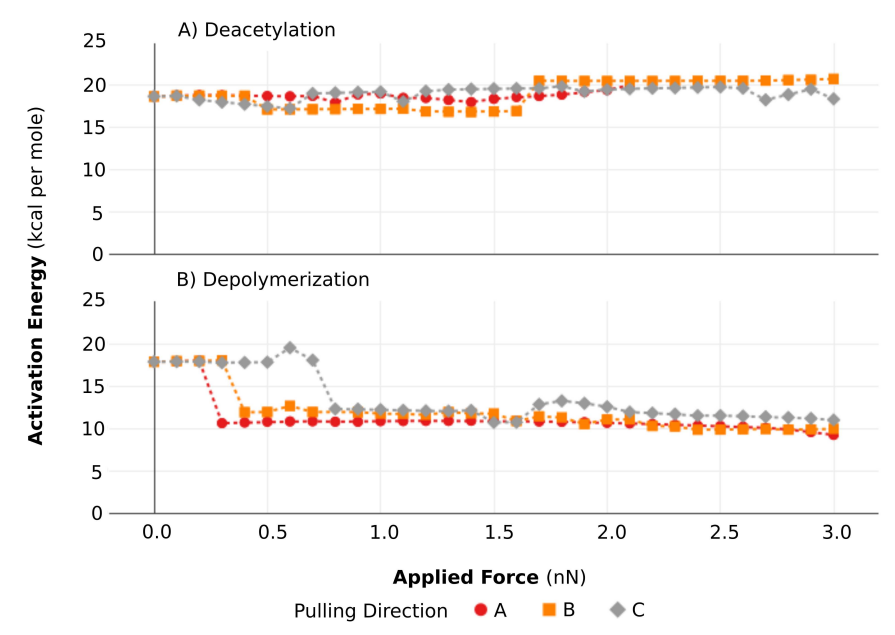

Figure 6: $E_{a}$ progression of GlcNAc (A) deacetylation and (B) depolymerization as a function of the applied force

The force modification algorithm was used to determine the optimized structures of the reactants and transition states in the RDSs of the deacetylation and depolymerization reactions. From the calculated $E_{a} s$ in FMPES, a sudden drop in $E_{a}$ by $8 \mathrm{kcal} \cdot \mathrm{mol}^{-1}$ was observed in the depolymerization, as shown in Figure 6, which suggests that the mechanochemical perturbation activated the depolymerization reaction. The effect of the pulling directions was manifested with the difference in the required force to induce the activation. The direction dependence of mechanochemical activation has been studied and proven in several experimental and theoretical studies. ${ }^{40-42}$ In addition to activation, it was also proven that it is possible to deactivate a reaction coordinate by controlling the direction of the force vector. ${ }^{43}$ The importance of the direction in this case is minor as the three directions all resulted in the activation of the reaction. In contrast, no characteristic change was observed in the $E_{a} s$ of the deacetylation RDS with the introduction of pulling forces. Hence, the deacetylation was found to be unsusceptible to the effect of mechanical pulling in these directions within the range of the applied pulling force. The selected force modified structures are appended in SI.

Notably, the induced difference in $E_{a} s$ also follows the experimentally observed results, that mechanical activation leads to depolymerization selective hydrolysis. ${ }^{11}$ To determine the 
origin of the sudden decrease in the activation energy of depolymerization, the energy changes of the reactant and transition states were compared. From Figure 7, it is evident that the depolymerization activation can be attributed to the reactant state destabilization. At the applied force of $0.3-0.8 \mathrm{nN}$, the energy of the reactant state became unstable discontinuously by approximately $6-8 \mathrm{kcal} \cdot \mathrm{mol}^{-1}$, while no such change was found in the transition state.

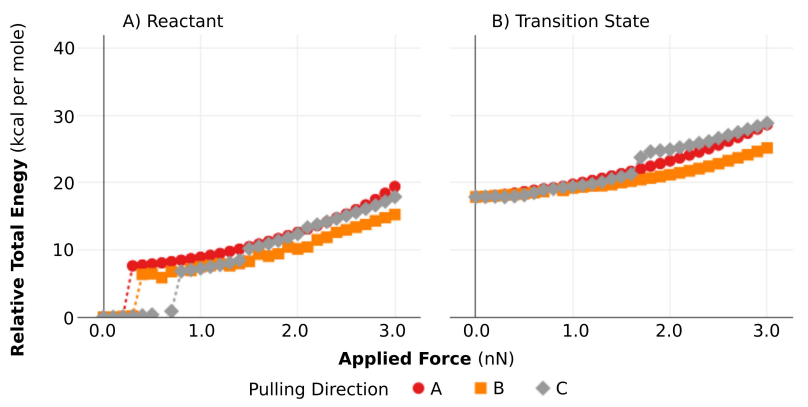

Figure 7: Relative energies of the depolymerization (A) reactant and (B) transition state as a function of the applied force

As the molecular geometric configuration dictates the energy, it is necessary to investigate the structural changes with respect to the relative energy along with the applied forces. To study the effect of the force in the location of the global minima in the hypersurfaces, root mean square displacements (RMSD) was chosen to quantitatively depict the structural progressions. The RMSD calculations were performed to compare two structures, $\mathrm{X}$ and $\mathrm{Y}$, with different applied forces, $\mathrm{x}$ and $\mathrm{y}$, respectively. $\mathrm{X}$ and $\mathrm{Y}$ can denote the reactant and transition states. The RMSD is defined as

$$
R M S D=\sqrt{\frac{|\Delta|^{2}}{N_{\text {atom }}}}
$$

where $\Delta=\mathbf{R}_{X, x}-\mathbf{R}_{Y, y} \cdot \mathbf{R}_{X, x}$ and $\mathbf{R}_{Y, y}$ are the atomic coordinates and $N_{\text {atom }}$ is the number of atoms included in the system. The RMSD is minimized by maximizing the overlap of the $\mathrm{X}$ and $\mathrm{Y}$ structures. This analysis visualizes the structure changes between the reactant states, transition states, and reactant-transition states with different applied forces, which is useful for determining the divergence or convergence of the two states as the applied force 
increases.

The RMSD matrix in Figure 8(a) shows the structural deviation among the reactant states with different external forces. A striking color change was observed at an applied force of $0.3 \mathrm{nN}$, which indicates the abrupt change in the structure of the reactant state at an applied force of $0.3 \mathrm{nN}$. In contrast, Figure $8(\mathrm{~b})$ shows the gradual change in the transition state structure with an increase in the applied force. These matrices reflected the energy changes in pulling direction A (Figure 7). Figure 8(c) summarizes the similarity between the reactant and transition state at different applied forces. It can be seen that the reactant structure approaches the transition state structure. The horizontal rectangular orange region below $0.3 \mathrm{nN}$ shows that the structures of the reactant states are less similar to those of the transition states at any applied force. In contrast, over $0.3 \mathrm{nN}$, the RMSD of the corresponding reactant and transition state structures start exhibiting structural similarity. As stated earlier, the reactant structure changes at this applied force. This follows the Hammond postulate, which states that the activation barrier for the reaction decreases when the similarity between the reactant and transition state structures increases. ${ }^{44,45}$ Similar trends were observed for pulling directions B and C, which are appended as Figures S4 and S5 in SI.

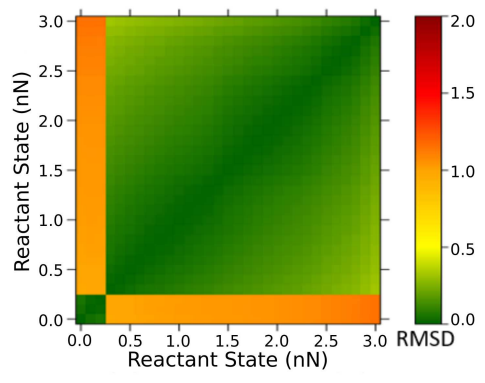

(a)

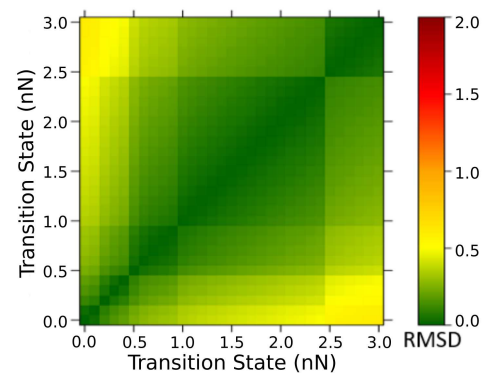

(b)

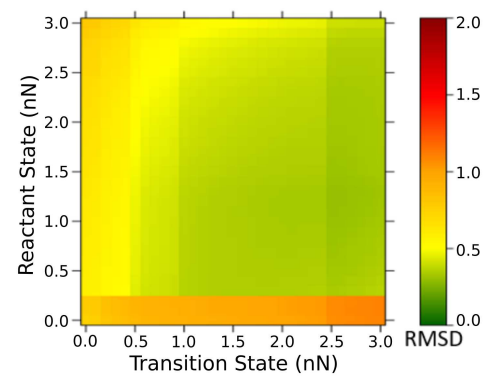

(c)

Figure 8: Depolymerization RMSD matrices for pulling direction A for (a) reactant-reactant (b) transition state-transition state and (c) reactant-transition state comparisons

The changes in the RMSD and energy are associated with the glycosidic dihedral angle, C5-O5-C1-O1 (Figure 9). The change in the dihedral angle was greater than $30^{\circ}$ for all 


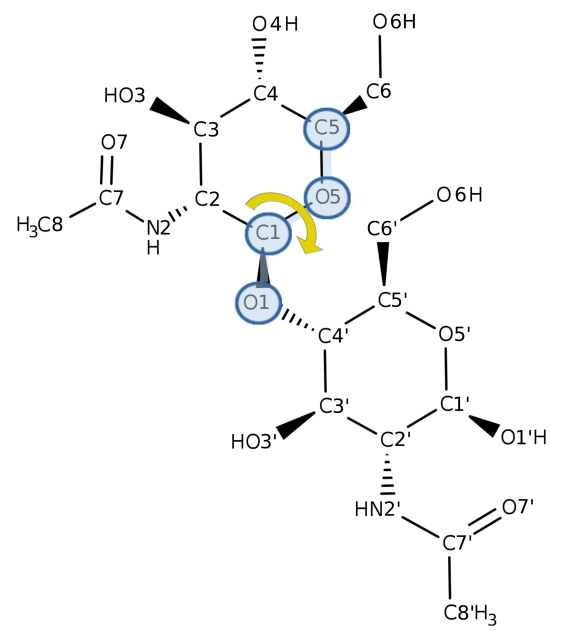

Figure 9: Mechanical force susceptible internal coordinate in the GlcNAc dimer

the pulling directions (Table 1). To confirm that the glycosidic dihedral angle is indeed the associated internal coordinate needed for the activation, constrained geometry optimizations with the C5-O5-C1-O1 angle fixed to $-94.3^{\circ}$ without external applied force were performed for the reactant and transition state. The calculated activation energy in this case was determined as $11.0 \mathrm{kcal} \cdot \mathrm{mol}^{-1}$, similar to those of the external force activated cases $(\sim 10$ $\left.\mathrm{kcal} \cdot \mathrm{mol}^{-1}\right)$.

It is also noted that the transition state structure was preserved during the introduction of the external forces. This is evidenced by the lower values of calculated RMSD values shown in Figure 8(b). Furthermore, the imaginary frequency varied minimally within computational accuracy. Thus, it can then be interpreted that the original intrinsic reaction coordinate was also preserved around the transition state.

Similar RMSD matrices for the deacetylation were also prepared, analyzed, and are shown in Figure S6 of SI. It should be noted that the diagonal elements of the RMSD matrix for the reactant-transition state remained similar, as shown in in Figure S6(c). This indicates that the structural differences of the reactant and transition state remain similar upon the application of a pulling force.

Energy decomposition analysis (EDA) was performed to account for the role of each 


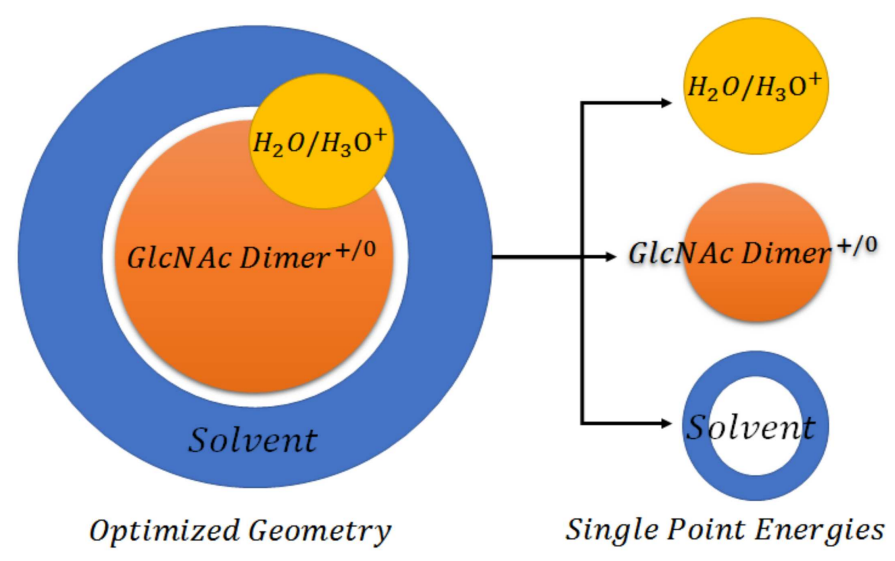

Figure 10: Energy decomposition scheme

fragment in the model. This also allows the determination of the behavior of stressed GlcNAc dimer with increasing force. The decomposition scheme is illustrated in Figure 10. The decomposition was conducted by separating different fragments in the chemical model. Specifically, this corresponds to 1) the GlcNAc dimer, 2) the attacking hydronium molecule, and 3) the solvent molecules.

Aside from the single point energies (SPE), the two-body interaction energy (IE) was calculated as

$$
I E_{A B}(F)=E_{A B}(F)-E_{A}(F)-E_{B}(F)
$$

where $\mathrm{E}$ is the total electronic energy, $\mathrm{F}$ is the applied external force, and $\mathrm{A}, \mathrm{B} \in\left\{\mathrm{H}_{2} \mathrm{O}, \mathrm{H}_{3} \mathrm{O}^{+}\right.$, GlcNAc dimer, Solvent\}

Analyzing the two-body interaction energies in the depolymerization reactant state, it was found that the trend in the total electronic energy follows that of the GlcNAc-solvent interaction (Figure S7). This change can be attributed to the conformational change in the GlcNAc dimer which disrupted the orientation of the water cluster, effectively changing the solvent interaction.

As the change in the depolymerization activation energy is dictated by the reactant destabilization due to the change in the GlcNAc-solvent interaction, the calculated change in $E_{a}$ could be affected by the solvent model used in the study. However, the EDA result for 


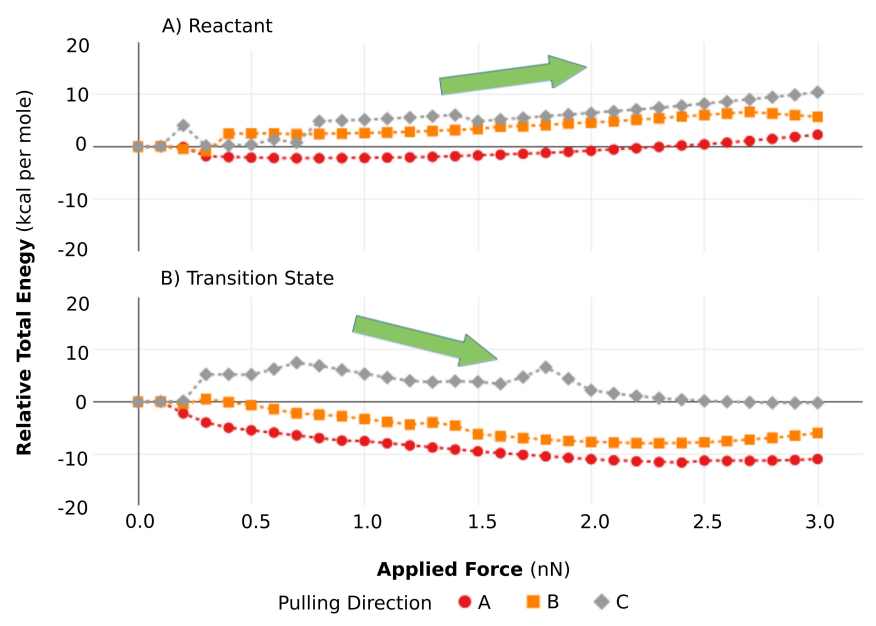

Figure 11: Selected energies in the decomposition analysis. Single point energy of the GlcNAc dimer in the (A) reactants and (B) transition states of the depolymerization as a function of the external force

the GlcNAc dimer fragment (Figure 11) shows that the energy of the reactant state increases with the applied force, while that of the transition state decreases. Hence, the activation of the depolymerization also originates from the energy change in the GlcNAc dimer fragment. Considering only the GlcNAc fragment, the activation energies as function of applied forces (F) were recalculated as

$$
E_{a}(F)=E(F)_{\text {GlcNAcDimer }}^{\text {TS }}-E(F)_{\text {GlcNAcDimer }}^{\text {Reactant }}
$$

Figure 12 shows that the GlcNAc fragment is intrinsically activated upon pulling along all the sampled directions.

In the case of the deacetylation, the result of EDA revealed that the energy contribution of the GlcNAc dimer increases with the magnitude of the applied force, as shown in Figure S8. The amount of increase in the reactant state is close to that in the transition state. The structural analysis in Figure S6 also shows that the structural difference between the reactant and the transition state remains the same. This verifies that the deacetylation reaction, within the limits of the sampled forces in this research, is insusceptible to either mechanical activation or deactivation. 


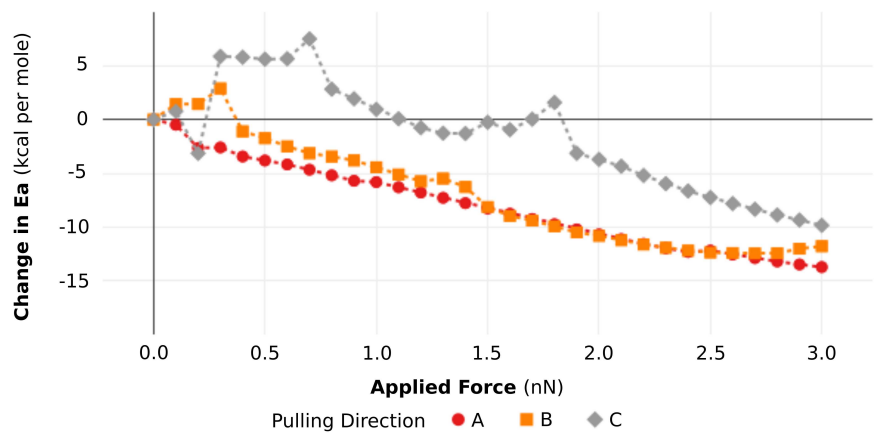

Figure 12: Change in the activation energy with an applied force in the GlcNAc fragment.

The electronic structure of the active sites were then analyzed via NBO calculations. ${ }^{29}$ As previously mentioned, in the RDS of the deacetylation reaction, the active site is the carbonyl carbon of the $N$-acetyl arm of the GlcNAc dimer, while that of the depolymerization reaction is the glycosidic oxygen of the same molecule. Using the second order perturbation theory analysis, the stabilization due to the electron delocalization among the NBOs was calculated. In this paradigm, $\mathrm{E}(2)$ is the associated stabilization energy when an electron is donated from an occupied NBO to an empty NBO. We note that $\mathrm{E}(2)$ is a measure of the orbital interaction and does not directly interpret the destabilization of the reactant state under the applied force.

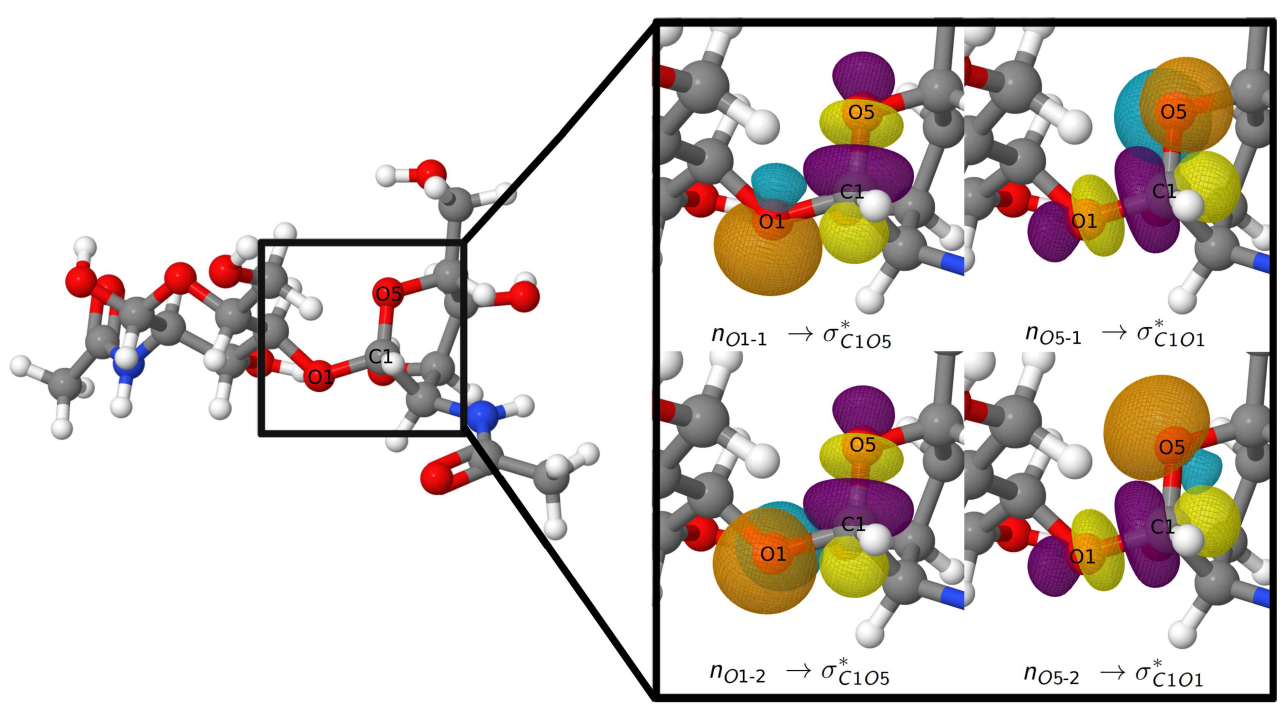

Figure 13: Selected donor-acceptor natural bonding orbitals of the GlcNAc dimer in the relaxed reactant state of the depolymerization 
In the case of the deacetylation, there was no significant change in the electron delocalization in the NBO basis. The selected donor-acceptor, orbital occupancy, and partial charges for the deacetylation are summarized in Tables S1 and S2 in SI. This was expected, as the local geometry in the model was maintained under the applied force. Consequently, the orbital occupancy and partial charges remained similar to the relaxed reactant state which corresponds to the unsusceptibility of the deacetylation to mechanical activation-deactivation in the range of the sampled applied forces.

In contrast, the depolymerization case, which is activated upon the application of an external force, showed a significant change in the electron donor-acceptor interaction on the onset of activation. In Table 1 , the interactions concerning the glycosidic bond and the depolymerization active site are tabulated with orbital naming following that in Figure $13 .{ }^{46}$

Table 1: Stabilizing interaction $\mathrm{E}(2)\left(\mathrm{kcal} \cdot \mathrm{mol}^{-1}\right)$ for the depolymerization reactant state at different directions and forces

\begin{tabular}{|c|c|c|c|c|c|c|c|c|c|}
\hline \multirow{2}{*}{$\begin{array}{c}\text { Pulling } \\
\text { Direction }\end{array}$} & \multicolumn{2}{|c|}{ Interaction } & \multicolumn{7}{|c|}{ Applied Force (nN) } \\
\hline & Donor & Acceptor & 0.00 & 0.50 & 1.00 & 1.50 & 2.00 & 2.50 & 3.00 \\
\hline \multirow{5}{*}{$\mathrm{A}$} & $n_{O 1-1}$ & $\sigma_{C 1 O 5}^{*}$ & 3.19 & 2.34 & 2.37 & 2.57 & 2.69 & 2.69 & 2.75 \\
\hline & $n_{O 1-2}$ & $\sigma_{C 1 O 5}^{*}$ & 2.22 & 4.88 & 5.00 & 5.07 & 5.01 & 5.22 & 5.52 \\
\hline & $n_{O 5-1}$ & $\sigma_{C 1 O 1}^{*}$ & 2.96 & $<0.50$ & $<0.50$ & $<0.50$ & $<0.50$ & $<0.50$ & $<0.50$ \\
\hline & $n_{O 5-2}$ & $\sigma_{C 1 O 1}^{*}$ & 3.47 & 18.39 & 18.27 & 18.03 & 17.76 & 17.36 & 16.55 \\
\hline & Dihedr & l angle ${ }^{a}$ & -155.9 & -94.3 & -96.2 & -98.4 & -100.6 & -103.2 & -107.3 \\
\hline \multirow{5}{*}{$\mathrm{B}$} & $n_{O 1-1}$ & $\sigma_{C 1 O 5}^{*}$ & 3.19 & 1.06 & 0.81 & 1.76 & 1.53 & $<0.50$ & 4.44 \\
\hline & $n_{O 1-2}$ & $\sigma_{C 1 O 5}^{*}$ & 2.22 & 4.19 & 4.78 & 5.01 & 5.65 & 6.16 & 0.62 \\
\hline & $n_{O 5-1}$ & $\sigma_{C 1 O 1}^{*}$ & 2.96 & $<0.50$ & $<0.50$ & $<0.50$ & $<0.50$ & $<0.50$ & $<0.50$ \\
\hline & $n_{O 5-2}$ & $\sigma_{C 1 O 1}^{*}$ & 3.47 & 14.63 & 14.74 & 14.40 & 14.15 & 15.20 & 17.59 \\
\hline & Dihedr & l angle ${ }^{a}$ & -155.9 & -120.6 & -120.3 & -118.6 & -119.5 & -119.01 & -93.9 \\
\hline \multirow{5}{*}{$\mathrm{C}$} & $n_{O 1-1}$ & $\sigma_{C 1 O 5}^{*}$ & 3.19 & 2.70 & 1.22 & 0.85 & 0.73 & 0.61 & 0.53 \\
\hline & $n_{O 1-2}$ & $\sigma_{C 1 O 5}^{*}$ & 2.22 & 2.82 & 3.34 & 3.65 & 3.64 & 3.76 & 3.70 \\
\hline & $n_{O 5-1}$ & $\sigma_{C 1 O 1}^{*}$ & 2.96 & 2.72 & 0.64 & $<0.50$ & $<0.50$ & $<0.50$ & $<0.50$ \\
\hline & $n_{O 5-2}$ & $\sigma_{C 1 O 1}^{*}$ & 3.47 & 4.21 & 13.64 & 18.47 & 18.99 & 19.72 & 19.96 \\
\hline & \multicolumn{2}{|c|}{ Dihedral angle ${ }^{a}$} & -155.9 & -153.3 & -125.3 & -107.2 & -105.9 & -102.0 & -102.2 \\
\hline
\end{tabular}

${ }^{a}$ Dihedral angle, C5-O5-C1-O1, in degrees. See Figure 9 for atomic labels.

On the onset of activation, which is also marked by the change in the dihedral angle 
highlighted in blue in Figure 9, there is a corresponding significant change in the stabilizing E(2) energy. Table 1 shows that the conformational change due to the mechanical force accompanies electron donation from the non-bonding lone pair $n_{O 5-2}$ orbital to the glycosidic anti-bonding $\sigma_{C 1 O 1}^{*}$ orbital (See Figure 13). For example, in pulling direction A, the E(2) for $n_{O 5-2}-\sigma_{C 1 O 1}^{*}$ shows a discontinuous increase from $3.47 \mathrm{kcal} \cdot \mathrm{mol}^{-1}$ to $18.39 \mathrm{kcal} \cdot \mathrm{mol}^{-1}$ when the applied force changes from $0.0 \mathrm{nN}$ to $0.5 \mathrm{nN}$. The same trend is followed for the other pulling directions. Hence, the activation can be attributed to this donor-acceptor interaction.

Table 2: Selected orbital occupancy for the depolymerization reactant state at different pulling directions and forces

\begin{tabular}{ccccccccc}
\hline \multirow{2}{*}{$\begin{array}{c}\text { Pulling } \\
\text { Direction }\end{array}$} & Orbital & & \multicolumn{7}{c}{ AppliedForce $(\mathrm{nN})$} \\
\cline { 3 - 9 } & & 0.00 & 0.50 & 1.00 & 1.50 & 2.00 & 2.50 & 3.00 \\
\hline \multirow{2}{*}{$\mathrm{A}$} & $\sigma_{C 1 O 1}^{*}$ & 0.048 & 0.085 & 0.085 & 0.085 & 0.084 & 0.083 & 0.081 \\
& $\sigma_{C 1 O 5}^{*}$ & 0.036 & 0.037 & 0.041 & 0.048 & 0.042 & 0.042 & 0.043 \\
\hline \multirow{2}{*}{$\mathrm{B}$} & $\sigma_{C 1 O 1}^{*}$ & 0.048 & 0.077 & 0.078 & 0.075 & 0.075 & 0.082 & 0.079 \\
& $\sigma_{C 1 O 5}^{*}$ & 0.036 & 0.036 & 0.036 & 0.039 & 0.040 & 0.038 & 0.041 \\
\hline \multirow{2}{*}{$\mathrm{C}$} & $\sigma_{C 1 O 1}^{*}$ & 0.048 & 0.050 & 0.075 & 0.089 & 0.092 & 0.095 & 0.096 \\
& $\sigma_{C 1 O 5}^{*}$ & 0.036 & 0.036 & 0.035 & 0.034 & 0.033 & 0.033 & 0.033 \\
\hline
\end{tabular}

To further probe the changes in the electronic structure, the orbital occupancies along the glycosidic bond are summarized in Table 2. For the depolymerization reaction in which an abrupt change was observed in the stabilizing energy $E(2)$, an expected increase in the occupancy of the $\sigma_{C 1 O 1}^{*}$ orbital was also observed. In the case of pulling direction A, the occupancy of $\sigma_{C 1 O 1}^{*}$ increases at the applied force of $0.5 \mathrm{nN}$, while no such change was found in that of $\sigma_{C 1 O 5}^{*}$. The same trend was observed in other pulling directions. The main contributing donor-acceptor interaction can then be inferred to be $n_{O 5-2} \rightarrow \sigma_{C 1 O 1}^{*}$ (See Table 1).

Upon the application of a limiting force in a pulling manner, the reactant structure was destabilized, and the $\sigma_{C 1 O 1}^{*}$ occupancy was increased. In our result, the occupancy of $\sigma_{C 1 O 1}^{*}$ increased by a factor of $>1.5$ in all pulling directions. Since there is an increase in the 
occupancy of the said anti-bonding orbital, the corresponding glycosidic bond decreases its bond order, rendering this bond weaker. As these suggest that the electron density in the active site is increased, an increase in the Brønsted-Lowry basicity of the glycosidic oxygen is expected as a consequence.

To assess the change in the Brønsted-Lowry basicity, the relative electrostatic interaction energy $(\Delta E)$ of the glycosidic oxygen with a point charge $(\mathrm{q}=+1)$ at the typical hydrogen bonding distance of $2 \AA$ was calculated as

$$
\Delta E=\frac{(+1) \Delta Q}{r}
$$

where $r=2 \AA$ and $\Delta Q$ is the NBO partial charge difference of the stressed and relaxed $(F=0)$ structures in the reactant state. This is a reasonable measure because hydrogen bonding originates from electrostatic interactions. ${ }^{47}$

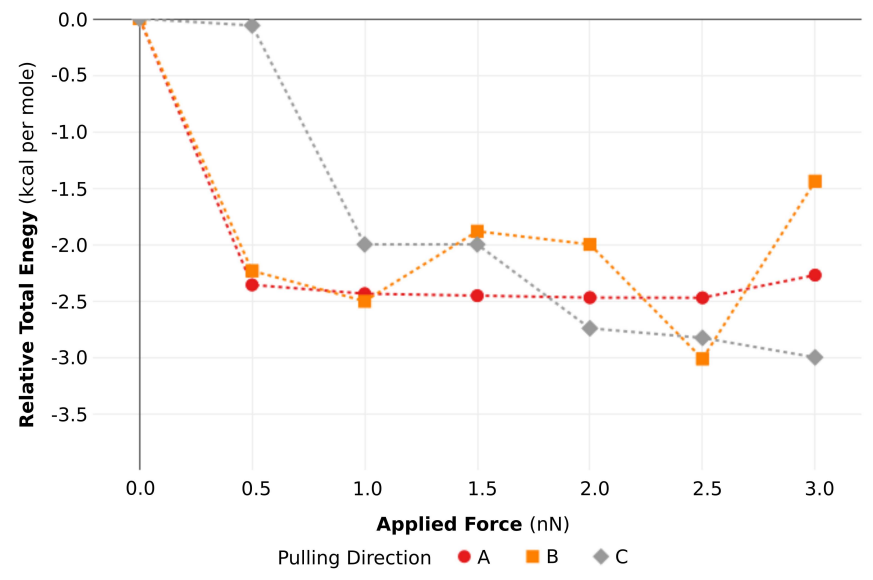

Figure 14: Relative energy associated with the electrostatic interaction with increasing applied force. See Eq. 4 for the definition.

Figure 14 summarizes the electrostatic interaction calculated according to Eq. 4. A significant increase in the electrostatic interaction up to $-3 \mathrm{kcal} \cdot \mathrm{mol}^{-1}$ was found with the application of external force, which is consistent with the changes in the second order energy in Table 1 and the orbital occupancy in Table 2. Thus, it can be concluded that the basicity 
of the active site was increased; consequently, the proton affinity and, therefore, the reactivity were also increased. As the partial negativity of the glycosidic oxygen increases, the protonation should be relatively easier due to the electronic structural change caused by the applied force. It is also known that the other hydroxyl oxygen sites of the GlcNAc dimer are more basic than the glycosidic oxygen; hence, these sites compete for the proton at low acid concentrations. ${ }^{6}$ Thus, this increase in basicity is important for the acid catalysis to occur.

\section{Conclusions}

The electronic structural effect of mechanochemical pulling, on the competing chitin hydrolysis reactions was investigated by DFT calculations with an externally applied force. In the calculations without force, the RDSs of deacetylation and depolymerization have similar activation energies, which reflect the non-selective acid hydrolysis observed experimentally. ${ }^{1}$

The mechanical pulling exerted different effects on the competing reactions. In the deacetylation reactions, the energies of the reactant and transition state increased monotonically, resulting in an almost constant deacetylation activation energy with increasing applied force up to $3 \mathrm{nN}$. In the mechanistic studies, the RDS of deacetylation is the nucleophilic attack of $\mathrm{H} 2 \mathrm{O}$, not the $\mathrm{C}-\mathrm{N}$ bond cleavage. This is one of the reasons why the deacetylation is insusceptible to the external pulling. Contrastingly, the depolymerization activation energy significantly decreased with the application of sufficient pulling force with respect to the sampled directions. 


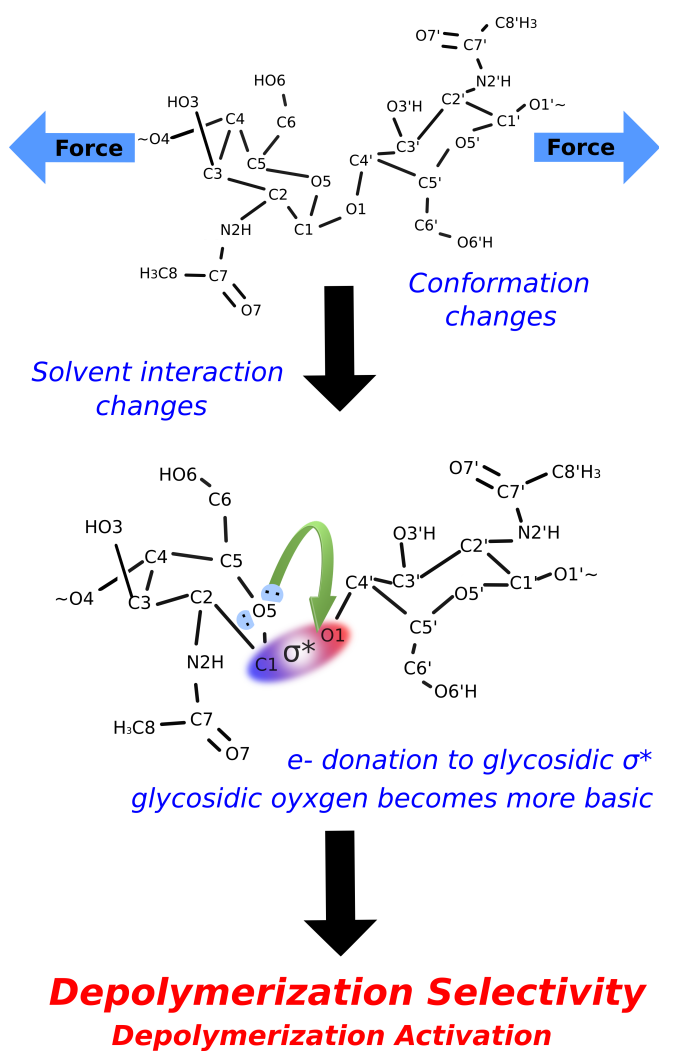

Figure 15: Scheme of mechanochemical activation toward chitin depolymerization

The activation of depolymerization can be mainly attributed to the destabilization of the reactant state upon the introduction of sufficient pulling force. A graphical representation of the activation of depolymerization is shown in Figure 15. The mechanical stress changes the GlcNAc dimer conformation. In the depolymerization active site, the glycosidic dihedral angle, C5-O5-C1-O1, rotates by more than 30 degrees which induces a syn-periplanar conformation of an $\mathrm{O} 5$ lone pair to the C1-O1 bond pair. This results in a significant increase in the charge transfer interaction from the $\mathrm{O} 5$ lone pair $\left(n_{O 5-2}\right)$ to the glycosidic bond anti-bonding orbital $\left(\sigma_{C 1 O 1}^{*}\right), n_{O 5-2} \rightarrow \sigma_{C 1 O 1}^{*}$, leading to a higher occupancy of the $\sigma_{C 1 O 1}^{*}$ orbital. Partial negative charges were found to increase at the glycosidic oxygen increasing its Brønsted-Lowry basicity, which facilitates the acid catalysis.

The results of this study showed that a mechanochemical pulling simulation, which represents a part of complex mechanical forces, can successfully interpret the selectivity enhance- 
ment of depolymerization. It should be noted, however, that the complexity addressed in this work revolves in the geometric deformation due to the mechanical pulling forces while neglecting the other components of mechanical force and instantaneous finite temperature effects associated with ball milling.

\section{Supporting Information Available}

Additional details for the FMPES implementation in G09. Force vector diagram of a ball bombarding to a molecular plane. Selected optimized structures for the deacetylation and depolymerization mechanistic studies. Selected optimized structures for the RMSD matrices for the depolymerization pulling simulations (Pulling Directions B and C) and the deacetylation pulling simulations (Pulling Direction B). GlcNAc dimer IE as function of the applied force. Energy decomposition analysis and NBO analysis for the deacetylation reaction.

\section{Acknowledgement}

This work was financially supported by the Japan Science and Technology Agency (JST) ALCA (Project No. JPMJAL1309), Japan Society for the Promotion of Science (JSPS) KAKENHI Grant-in-Aid for Scientific Research (B) (Nos. JP20H02685 and JP18H01781), and Ministry of Education, Culture, Sports, Science and Technology (MEXT) Project (Integrated Research Consortium on Chemical Sciences). This research was also partly supported by the Photo-excitonix Project from Hokkaido University. Part of the computations were carried out at RCCS (Okazaki, Japan), ACCMS (Kyoto University), and RIIT (Kyushu University). 


\section{References}

(1) Kaya, M.; Baran, T.; Karaarslan, M. A new method for fast chitin extraction from shells of crab, crayfish and shrimp. Nat. Prod. Res. 2015, 29, 1477-1480.

(2) Percot, A.; Viton, C.; Domard, A. Optimization of chitin extraction from shrimp shells. Biomacromolecules 2003, 4, 12-18.

(3) Arbia, W.; Arbia, L.; Adour, L.; Amrane, A. Chitin extraction from crustacean shells using biological methods -A review. Food Technol. Biotechnol. 2013, 51, 12-25.

(4) Hamdi, M.; Hammami, A.; Hajji, S.; Jridi, M.; Nasri, M.; Nasri, R. Chitin extraction from blue crab (Portunus segnis) and shrimp (Penaeus kerathurus) shells using digestive alkaline proteases from P. segnis viscera. Int. J. Biol. Macromol. 2017, 101, 455-463.

(5) Younes, I.; Hajji, S.; Frachet, V.; Rinaudo, M.; Jellouli, K.; Nasri, M. Chitin extraction from shrimp shell using enzymatic treatment. Antitumor, antioxidant and antimicrobial activities of chitosan. Int. J. Biol. Macromol. 2014, 69, 489-498.

(6) Einbu, A.; Vrum, K. M. Depolymerization and de-N-acetylation of chitin oligomers in hydrochloric acid. Biomacromolecules 2007, 8, 309-314.

(7) Chen, X.; Yan, N. Novel catalytic systems to convert chitin and lignin into valuable chemicals. Catal. Surv. Asia 2014, 18, 164-176.

(8) Konopka, J. B. N-acetylglucosamine (GlcNAc) functions in cell signaling. Scientifica 2012, 2012, 489208-48922.

(9) Shikhman, A. R.; Kuhn, K.; Alaaeddine, N.; Lotz, M. N-acetylglucosamine prevents IL-1B-mediated activation of human chondrocytes. J. Immunol. 2018, 1-15.

(10) Wasonga, G.; Tatara, Y.; Kakizaki, I.; Huang, X. Synthesis of N -acetyl glucosamine analogs as inhibitors for hyaluronan biosynthesis. J. Carbohydr. Chem. 2013, 32, 392409. 
(11) Yabushita, M.; Kobayashi, H.; Kuroki, K.; Ito, S.; Fukuoka, A. Catalytic depolymerization of chitin with retention of N-acetyl group. ChemSusChem 2015, 8, 3760-3763.

(12) Patel, D.; Marx, D.; East, A. Improving yield and rate of acid-catalyzed deconstruction of lignin by mechanochemical activation. ChemPhysChem 2020, 21.

(13) Ribas-Arino, J.; Marx, D. Covalent mechanochemistry: Theoretical concepts and computational tools with applications to molecular nanomechanics. Chem. Rev. 2012, 112, $5412-5487$.

(14) Stauch, T.; Dreuw, A. Advances in quantum mechanochemistry: electronic structure methods and force analysis. Chem. Rev. 2016, 116, 14137-14180.

(15) Binnig, G.; Quate, C. F.; Gerber, C. Atomic force microscope. Phys. Rev. Lett. 1986, 56, 930-933.

(16) De Bo, G. Mechanochemistry of the mechanical bond. Chem. Sci. 2018, 9, 15-21.

(17) Beyer, M. K. The mechanical strength of a covalent bond calculated by density functional theory. J. Chem. Phys. 2000, 112, 7307-7312.

(18) Ong, M. T.; Leiding, J.; Tao, H.; Virshup, A. M.; Martínez, T. J. First principles dynamics and minimum energy pathways for mechanochemical ring opening of cyclobutene. J. Am. Chem. Soc. 2009, 131, 6377-6379.

(19) Subramanian, G.; Mathew, N.; Leiding, J. A generalized force-modified potential energy surface for mechanochemical simulations. J. Chem. Phys. 2015, 143, 134109.

(20) Ribas-Arino, J.; Shiga, M.; Marx, D. Understanding covalent mechanochemistry. Angew. Chem., Int. Ed. 2009, 48, 4190-4193.

(21) Wolinski, K.; Baker, J. Theoretical predictions of enforced structural changes in molecules. Mol. Phys. 2009, 107, 2403-2417. 
(22) Becke, A. D. Density-functional thermochemistry. III. The role of exact exchange. J. Chem. Phys. 1993, 98, 5648-5652.

(23) Yamabe, S.; Guan, W.; Sakaki, S. Three competitive transition states at the glycosidic bond of sucrose in its acid-catalyzed hydrolysis. J. Org. Chem. 2013, 78, 2527-2533.

(24) De Chavez, D. P.; Gao, M.; Kobayashi, H.; Fukuoka, A.; Hasegawa, J. Adsorption mediated tandem acid catalyzed cellulose hydrolysis by ortho-substituted benzoic acids. Mol. Catal. 2019, 475, 110459.

(25) Miertuš, S.; Scrocco, E.; Tomasi, J. Electrostatic interaction of a solute with a continuum. A direct utilizaion of $\mathrm{AB}$ initio molecular potentials for the prevision of solvent effects. Chem. Phy. 1981, 55, $117-129$.

(26) Mennucci, B.; Tomasi, J. Continuum solvation models: A new approach to the problem of solute's charge distribution and cavity boundaries. J. Chem. Phys. 1997, 106, 51515158.

(27) Cammi, R.; Mennucci, B.; Tomasi, J. Fast evaluation of geometries and properties of excited molecules in solution: a Tamm-Dancoff model with application to 4dimethylaminobenzonitrile. J. Phys. Chem. A 2000, 104, 5631-5637.

(28) Fukui, K. The path of chemical reactions - the IRC approach. Acc. Chem. Res. 1981, $14,363-368$.

(29) Glendening, E. D.; Reed, A. E.; Carpenter, J. E.; Weinhold, F. NBO Version 3.1. 2011.

(30) Foster, J. P.; Weinhold, F. Natural hybrid orbitals. J. Am. Chem. Soc. 1980, 102, 7211-7218.

(31) Reed, A. E.; Weinhold, F. Natural bond orbital analysis of near-Hartree-Fock water dimer. J. Chem. Phys. 1983, 78, 4066-4073. 
(32) Reed, A. E.; Weinstock, R. B.; Weinhold, F. Natural population analysis. J. Chem. Phys. 1985, 83, 735-746.

(33) Reed, A. E.; Curtiss, L. A.; Weinhold, F. Intermolecular interactions from a natural bond orbital, donor-acceptor viewpoint. Chem. Rev. 1988, 88, 899-926.

(34) Carpenter, J.; Weinhold, F. Analysis of the geometry of the hydroxymethyl radical by the "different hybrids for different spins" natural bond orbital procedure. J. Mol. Struct.: THEOCHEM 1988, 169, $41-62$.

(35) Margoutidis, G.; Parsons, V. H.; Bottaro, C. S.; Yan, N.; Kerton, F. M. Mechanochemical amorphization of $\alpha$-chitin and conversion into oligomers of N-acetyl-d-glucosamine. ACS Sustainable Chem. Eng. 2018, 6, 1662-1669.

(36) Frisch, M. J.; Trucks, G. W.; Schlegel, H. B.; Scuseria, G. E.; Robb, M. A.; Cheeseman, J. R.; Scalmani, G.; Barone, V.; Mennucci, B.; Petersson, G. A. et al. Gaussian09 Revision E.01. Gaussian Inc.: Wallingford, CT, 2009.

(37) Zahn, D.; Schmidt, K. F.; Kast, S. M.; Brickmann, J. Quantum/classical investigation of amide protonation in aqueous solution. J. Phys. Chem. A 2002, 106, 7807-7812.

(38) Zahn, D. Theoretical study of the mechanisms of acid-catalyzed amide hydrolysis in aqueous solution. J. Phys. Chem. B 2003, 10\%, 12303-12306.

(39) Liang, X.; Montoya, A.; Haynes, B. S. Local site selectivity and conformational structures in the glycosidic bond scission of cellobiose. J. Phys. Chem. B 2011, 115, 1068210691.

(40) Lin, Y.; Barbee, M. H.; Chang, C.-C.; Craig, S. L. Regiochemical effects on mechanophore activation in bulk materials. J. Am. Chem. Soc. 2018, 140, 1596915975. 
(41) Barbee, M. H.; Kouznetsova, T.; Barrett, S. L.; Gossweiler, G. R.; Lin, Y.; Rastogi, S. K.; Brittain, W. J.; Craig, S. L. Substituent effects and mechanism in a mechanochemical reaction. J. Am. Chem. Soc. 2018, 140, 12746-12750.

(42) Stauch, T.; Dreuw, A. Force-induced retro-click reaction of triazoles competes with adjacent single-bond rupture. Chem. Sci. 2017, 8, 5567-5575.

(43) Krupicka, M.; Marx, D. Disfavoring mechanochemical reactions by stress-induced steric hindrance. J. Chem. Theory Comput. 2015, 11, 841-846.

(44) Hammond, G. S. A correlation of reaction rates. J. Am. Chem. Soc. 1955, 77, 334-338.

(45) Konda, S. S. M.; Brantley, J. N.; Varghese, B. T.; Wiggins, K. M.; Bielawski, C. W.; Makarov, D. E. Molecular catch bonds and the anti-hammond effect in polymer mechanochemistry. J. Am. Chem. Soc. 2013, 135, 12722-12729.

(46) Loerbroks, C.; Rinaldi, R.; Thiel, W. The electronic nature of the 1,4-B-glycosidic bond and its chemical environment: DFT insights into cellulose chemistry. Chem. - Eur. J. 2013, 19, 16282-16294.

(47) Umeyama, H.; Morokuma, K. The origin of hydrogen bonding. An energy decomposition study. J. Am. Chem. Soc. 1977, 99, 1316-1332. 


\section{TOC Graphic}

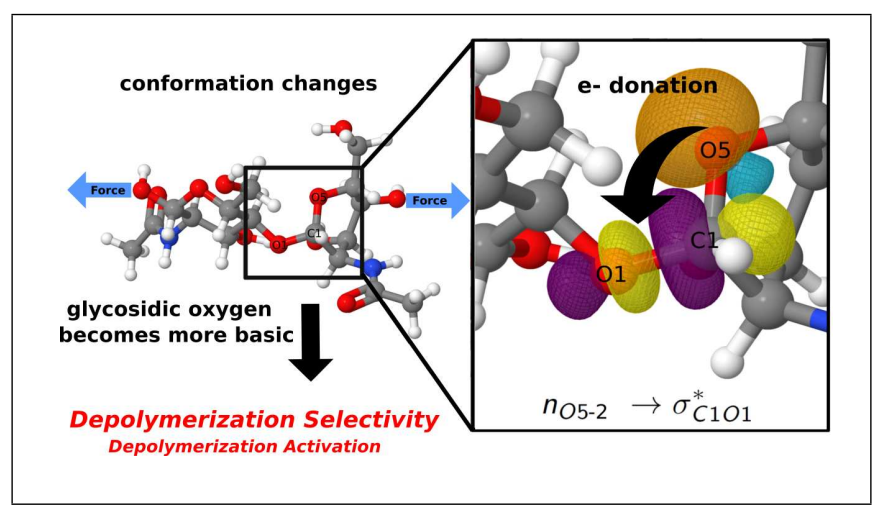

